\title{
EL ANÁLISIS DE LAS DIFERENCIAS EN EL PROCESO DE TRANSFERENCIA DE TECNOLOGÍA ENTRE REGIONES*
}

Henry Caicedo Asprilla**

* doi:10.11144/Javeriana.cao.31-56.adpt. El artículo se recibió el 31/01/2018 y se aprobó el 15/05/2018. Sugerencia de citación: Caicedo, H. (2018). El análisis de las diferencias en el proceso de transferencia de tecnología entre regiones. Cuadernos de Administración, 31(56), 163-194. http://dx.doi.org/10.11144/ Javeriana.cao.31-56.adpt

**Universidad del Valle, Cali, Colombia. E-mail: Henry.caicedo@correounivalle.edu.co 


\section{El análisis de las diferencias en el proceso de transferencia de tecnología entre regiones}

\section{RESUMEN}

Este artículo analiza la transferencia de tecnología entre regiones, con base en la teoría de redes sociales. Se aplicó el método del ARS a 137 regiones de diferente nivel de desarrollo, y se encontró que la transferencia de tecnología varía en dos sentidos: en uno lineal, en donde las regiones más desarrolladas, derraman conocimiento a las de menor nivel de desarrollo. Y en uno no lineal e interactivo, en donde las regiones desarrolladas y las de menor nivel de desarrollo intercambian conocimiento. También se verificó que existen regiones más transmisoras que receptoras, así como regiones más receptoras que transmisoras de tecnología. Se concluye que todas las regiones tienen algún conocimiento que ofrecer, pero también algún conocimiento que necesitan adquirir.

Palabras claves: Transferencia de tecnología, redes de innovación externalidades, empresas multinacionales. Códigos JEL: F23, $\mathrm{O}_{32}, \mathrm{O}_{3} 8$

\section{The analysis of the differences in the technology transfer process between regions}

\section{A análise das diferenças no processo de transferência de tecnologia entre regiões}

\section{ABSTRACT}

Based on the social network theory, this paper seeks to understand the transfer of technology between regions. The ARS method was applied to 137 regions with different levels of development, and it was found that the transfer of technology varies in two ways: in a linear way, where the most developed regions spill knowledge to those with a lower level of development. And in a non-linear and interactive way, where developed and less developed regions exchange knowledge. It was also verified that some regions are more transmitters of technology than receivers, whereas some others are more receivers than transmitters. It is concluded that all regions have some knowledge to offer, but some knowledge to acquire as well.

Keywords: Transfer of technology, innovation networks, spillovers, multinational firms. JEL Codes: $\mathrm{F}_{23}, \mathrm{O}_{32}, \mathrm{O}_{3} 8$

\section{ResUmo}

Este artigo analisa a transferência de tecnologia entre regiões, tomando como base a teoria de redes sociais. Aplicou-se o método do ARS a 137 regiões com diferente nível de desenvolvimento, e encontrou-se que a transferência de tecnologia varia em dois sentidos: em um sentido lineal, no qual as regiões mais desenvolvidas transferem conhecimento às de menor nível de desenvolvimento. E em um sentido não lineal e interativo, no qual as regiões desenvolvidas e as de menor nível de desenvolvimento partilham conhecimento. Também se verificou tanto a existência de regiões mais transmissoras de tecnologia que receptoras quanto regiões mais receptoras que transmissoras. Conclui-se que todas as regiões têm algum conhecimento para oferecer, mas também algum conhecimento que precisam adquirir.

Palavras chave: Transferência de tecnologia, redes de inovação, externalidades, empresas multinacionais. Códigos JEL: $\mathrm{F}_{23}, \mathrm{O}_{32}, \mathrm{O}_{3} 8$ 


\section{Introducción}

Con el auge de la globalización en la década de 1990, las regiones pasaron a ser actores importantes en la coordinación de la vida económica (Storper, 1995); pero dado el acelerado ritmo del cambio tecnológico, se ha hecho evidente que estas no son autosuficientes a la hora de generar todo el conocimiento y tecnología para producir y competir (Gertler y Levitte, 2003; Giudice, Carayannis y Maggioni, 2017).

El proceso internacional de transferencia de tecnología -PIT- entre regiones tomó lugar como un fenómeno relevante (Ruscheweyh, Deppe, Lohmann et ál., 2013), en donde las regiones son a su vez agentes transmisores y emisores de tecnología (Storper y Venables, 2004; Maskell, Bathelt y Malmberg, 2006); proceso en el que las instituciones, redes y spillovers juegan un papel determinante en la circulación del conocimiento, entre empresas, universidades, centros de investigación, investigadores y las demás instituciones que componen los Sistemas Regionales de Innovación -SRI- (Yi, Chen, Wang y Kafouros, 2015; Coenen, Asheim, Bugge y Herstad, 2017). De aquí que la fortaleza de los SRI se ha constituido en una señal para atraer y localizar inversión extranjera directa -IED-, mano de obra calificada y toda clase de conocimiento (Huggins, Izushi, Prokop y Thompson, 2014).

El papel de las regiones como receptoras o emisoras de tecnología varía en función de sus ventajas competitivas (Coenen et ál., 2017). Unas regiones son competitivas en virtud de su conocimiento, por la calidad de la mano de obra, los centros de investigación, o por los clústeres (Morgan, 2017); mientras que otras lo son solo por sus materias primas, el tamaño del mercado, su localización geográfica, la cantidad de mano de obra, etc. (Cantwell y Glac, 2004; Giudice et ál., 2017).

En el PIT basado en redes y spillovers, las empresas multinacionales -EMN- se localizan dependiendo de si pueden explotar mercados o conocimientos (Cantwell y Glac, 2004; Narula y Pineli, 2017). Las universidades buscan pares para establecer convenios con otras para generar nuevos conocimientos o para transferirles el ya desarrollado (Bradley, Hayter y Link, 2013). La mano de obra se mueve hacia regiones donde encuentra mejores oportunidades (Coenen et ál., 2017). Mientras que la disposición de las cadenas globales de valor en las regiones obedece a si son proveedoras de materias primas o de servicios intensivos en conocimiento (Gereffi, Humphrey y Sturgeon, 2005). 
El PIT se mueve entre dos alternativas. La primera y más tradicional es aquella en donde las regiones más desarrolladas buscan a las de menor nivel de desarrollo para explotar sus mercados, materias primas y transferirles tecnologías (Cantwell y Glac, 2004). En este caso, el PIT va en una dirección y genera spillovers unilaterales (Caves, 1974; Casas et ál., 2013; Albornoz y López, 2015). La otra alternativa del PIT ocurre entre las regiones más desarrolladas, en donde estas intercambian saberes, tecnologías, servicios intensivos en conocimiento y mano de obra calificada (Morgan, 2017). Este es un PIT interactivo que va en doble vía, donde los agentes locales e internacionales son receptores y emisores activos en la transferencia de conocimientos y se aprovechan los spillovers recíprocos (Schmidt y Sofka; 2009; Driffield y Love, 2005).

En la actualidad el PIT entre regiones está matizado por la relevancia y heterogeneidad de los territorios locales (Ruscheweyh et ál, 2013; Morgan, 2017); una mayor intensidad en los vínculos entre agentes del SRI (Coenen et ál., 2017), y una mayor circulación y diversificación de los conocimientos (Maskell et ál., 2006; Keeble y Wilkinson; 2017). En este sentido, el propósito de este trabajo es contribuir a la explicación de las diferencias en el PIT cuando las regiones son heterogéneas, los agentes se articulan en redes y aprovechan los spillovers. Los objetivos que guían esta investigación son, en primer lugar, caracterizar las diferencias del PIT cuando participan regiones de diferente nivel de desarrollo y, en segundo término, tipificar a las regiones como transmisoras y/o receptoras de tecnología y conocimientos.

En el siguiente apartado se plantea un marco de análisis de transferencia de tecnología, usando las categorías del análisis de redes sociales -ARS-. A renglón seguido, en el tercer apartado se plantea la metodología de redes; en este sentido, se clasificó como sujetos de las redes a las regiones calificadas como globales por el GaWC e intensivas en el conocimiento por el KWCI. Se aplicó el análisis de correspondencia simple, tomando como categorías las posiciones que ocupan las regiones en competitividad según la OCDE (2012); el GaWC (2016) y el KWCI (2014). Se clasificaron las regiones de primer, segundo y tercer nivel de desarrollo. Se concluye que el ARS es un buen método para entender el actual PIT. También se deduce que las regiones en vía de desarrollo tienen un mayor espacio para generar ganancias intercambiando tecnologías con las regiones de países de reciente industrialización, que con las regiones más desarrolladas; porque con los territorios emergentes pueden desarrollarse más procesos de cooperación en igualdad de condiciones. 


\section{El proceso internacional de transferencia de tecnología entre regiones}

La revisión de literatura arroja que el PIT entre regiones experimenta diferentes niveles de conectividad, distancia y cercanía, donde interviene la manera en que estas se posicionen, ya sea como agentes transmisores y/o receptores de tecnología. Así las cosas, aquí se plantea como hipótesis de trabajo que el PIT entre las regiones más desarrolladas tiene mayor conectividad y menor distancia que el PIT que se configura entre las regiones de diferentes niveles de desarrollo. También se defiende la idea de que el rol de una región como transmisora o receptora de tecnología está en relación directa con el nivel de cercanía que experimente con las demás regiones.

Bozeman (2000), en su modelo de efectividad contingente, plantea que el proceso internacional de transferencia de tecnología -PIT- involucra cinco dimensiones: las características del agente emisor, las del agente receptor, el objeto de transferencia, el ambiente o entorno de las relaciones y, por último, las particularidades de los medios de transmisión. Años más tarde, Bozeman y Boardman (2014) complementaron el modelo de efectividad contingente con la inclusión del valor público de la tecnología y su impacto en el lugar de localización de la IED.

Paralelo a este, otros cambios se han hecho evidentes en el objeto de transferencia, en donde además de las máquinas ha cobrado relevancia la transferencia del conocimiento en un sentido amplio, involucrando las tecnologías blandas, la mano de obra calificada, el arte de hacer negocios, entre otros aspectos (Maskell et ál., 2006; Narula y Pineli, 2017; Giudice et ál., 2017).

En el ámbito geográfico el cambio más significativo es la relevancia de las regiones, en lugar de los países (Storper, 1995; Coenen et ál., 2017). La OCDE (2012) clasifica a las regiones en función de la fuente de su competitividad en tres grupos: las impulsadas por la innovación, las que se fundamentan en la eficiencia y las que se apalancan en los recursos naturales.

En la perspectiva de resaltar el protagonismo de las regiones, autores como Sassen (2001) y Taylor (2004) han creado el ranking GaWC, en el que las regiones son clasificadas según la capacidad de atraer IED y dominar los flujos de comercio. Son alpha las ciudades que enlazan a todo el mundo; beta, las que enlazan al continente de localización con el resto del mundo; gamma, las ciudades que integran al país con el continente; finalmente, son suficientes las regiones que solo enlazan al país y escasamente a países cercanos o claves. 
Por otra parte, Huggins e Izushi (2008) han planteado que la competitividad de una región se debe al uso que hacen del conocimiento; para medir este aspecto, crearon el KWCI. En esta lógica, Huggins et ál. (2014) clasificaron a las regiones en cinco grupos: líderes, seguidoras, emergentes, en transición y rezagadas. El orden viene dado por la fortaleza de su Sistemas Regionales de Innovación -SRI- (Boschma, 2014).

En los medios de transferencia, la transformación más significativa es la importancia que las redes han ido ganando como mecanismo de circulación de conocimiento (Carayannis, Campbell, Grigoroudis y Monteiro, 2017). Pero el cambio más profundo se asocia a la direccionalidad de los flujos de conocimientos, donde además del derrame o spillovers derivados de la tecnología que transfieren las regiones más desarrolladas a las de menor desarrollo, han cobrado especial interés los spillovers recíprocos, que operan cuando las regiones de mayor desarrollo aprenden y absorben tecnología y conocimiento de las menos desarrolladas (Driffield y Love, 2005; Schmidt y Sofka, 2009).

\section{La dinámica del PIT en red y spillovers entre regiones}

Reconocidos los cambios en el PIT, se hace necesario desarrollar un trabajo que explique cómo opera este proceso entre regiones en presencia de redes y spillovers. Este trabajo se inscribe en la literatura que investiga el papel de los agentes locales en el acceso a la transferencia de tecnología y aprovechamiento de las externalidades técnicas (Yi et ál., 2015; Narula y Pineli, 2017).

Valga decir que, en esta línea de investigación se identifican estudios que evalúan el impacto de la IED sobre los disímiles índices de productividad de las diferentes regiones en China resultantes de la heterogeneidad de las instituciones regionales (Yi et ál., 2015). Así mismo, aquellos que analizan los determinantes de la generación de spillovers en economías locales (Narula y Pineli, 2017) y la generación de conocimiento interno y externo en las firmas (Carayannis et ál., 2017).

Aquí se avanza en el análisis de la transferencia de tecnología entre regiones cuando estas son a su vez receptoras y transmisoras de mercados, comercio y personas, en el sentido de Sassen (2001); o reservorios de circulación de conocimientos (Huggins et ál., 2014); que pueden albergar firmas de tecnologías duras y blandas (Giudice et ál., 2017); atraer mano de obra para trabajar, divertirse y hacer negocios (Coenen et ál., 2017); y donde las universidades son referente de innovación y fuentes de conocimiento, 
con un importante papel en la producción de patentes (Bradley et ál., 2013); son lo que Caicedo (2017) denomina regiones globales basadas en el conocimiento.

El presente trabajo se apoya en el marco de análisis de redes sociales -ARS-; técnica muy útil para aquellas situaciones donde se constata la presencia de sujetos emisores y receptores y la presencia de vínculos entre ellos (Hanneman, 2000). En ese orden de ideas, aquí se asume que las regiones son sujetos en las redes de transferencia, en tanto que como contenedoras de sistemas de innovación — compuestos por empresas, universidades, instituciones financieras, centros de desarrollo tecnológicos, organismos públicos de apoyo a la investigación-, y dado que estos se comportan como un todo organizado, son a su vez receptoras y/o transmisoras de tecnología (Storper y Venables, 2004; Asheim, Boschma y Cooke, 2011; Yi et ál., 2015).

La OCDE (2012), Sassen (2016) y Huggins et ál. (2014) han argumentado, cada uno a su manera, que tienden a ser regiones transmisoras de tecnología aquellas que han logrado ser, respectivamente, innovadoras, alpha o beta, y líderes en la economía del conocimiento. Mientras que tienden a ser receptoras netas de tecnología las regiones que basan su competitividad en recursos naturales, las suficientes en globalización y rezagadas en la economía del conocimiento.

Constituyen los vínculos de la red, la IED, la movilidad de la mano de obra para trabajar y hacer negocios, al igual que por el cambio de residencia y el turismo, la colaboración en la creación de patentes, el hecho de compartir licencias, la colaboración en las publicaciones, los congresos especializados, las ferias temáticas; , en fin, toda la gama de posibilidades de intercambio de conocimientos que se presentan entre las regiones (Storper y Venables, 2004; Maskell et ál., 2006; Giudice et ál., 2017).

Las relaciones o vínculos entre regiones son de doble vía en aquellos casos en que las universidades y o las EMN de las regiones transmisoras, aparte de derramar spillovers a las receptoras, son capaces de capturar spillovers recíprocos. Por el contrario, los vínculos van en una sola vía en los casos en que los agentes de las regiones transmisoras de tecnología derraman spillovers en los agentes de las regiones de llegada, pero no capturan conocimiento de las regiones de acogida.

Se tiene entonces que el PIT en presencia de redes y spillovers se mueve entre dos extremos. Uno en donde los vínculos van en doble vía, que aquí se denomina como $\mathrm{PIT}^{\prime} \mathrm{NL}$, recogiendo el que este proceso opera bajo la lógica del modelo no lineal de Kline y 
Rosenberg (1986). Mientras que, en el otro extremo, en el proceso de transferencia, los enlaces van en una sola dirección, por lo que se le llama PIT'L, ya que opera más la lógica del modelo lineal (Godin, 2006).

Ahora bien, si se acepta la idea de que el PIT puede analizarse mediante redes, entonces la dinámica de este proceso responde a la lógica de una red social. En este sentido, para analizar los dos tipos extremos del PIT que se configuran cuando intervienen regiones de distinto nivel de desarrollo, aquí se usan las propiedades de conectividad, distancia y cercanía como proxys de las diferencias entre ellas.

Hanneman (2000) plantea que una red tiene más conectividad que otra si experimenta mayor tamaño o complejidad, más densidad, proximidad y si cada agente en promedio posee más conexiones. En esa línea de análisis, una red es más equilibrada si tiene un mayor índice de transitividad y reciprocidad. Además, afirma que los agentes transfieren la información de una manera más rápida y eficaz entre menor sea la distancia geodésica y la fragmentación de la red, porque menor es el esfuerzo que deben realizar para establecer contacto con otros. Finalmente, señala que el ARS permite identificar el rol de los agentes en una red. Para ello los indicadores de cercanía (closeness), poder de centralidad (degree) e intermediación (betweenness) son muy pertinentes. Estos índices en su conjunto dan cuenta de la posición y las ventajas que tiene una región sobre otra.

Bajo esta lógica, una región es más transmisora que receptora de tecnología si posee un mayor índice de outcloseness, lo que indica que tiene más capacidad de influir sobre las que están más cerca. Igualmente, el rol de transmisora de tecnología de una región aumenta cuando posee un más alto outdegree, lo cual indica que la región es capaz de llegar a más regiones que el resto de sus competidoras. De igual forma, un mayor índice de betweenness, o intermediación, refleja el hecho de que la región sirve de puente para enlazar a otras. Lo contrario también es cierto, una región es más receptora que transmisora de tecnología cuando experimenta mayor incloseness, indegree y betweenness. En esa perspectiva, un agente es más transmisor si tiene un mayor índice de cercanía, poder e intermediación que los demás. Lo contrario también es cierto, un agente es más receptor entre menores sean estos mismos índices (Hanneman, 2000).

Para analizar las diferencias en el PIT entre regiones en presencia de redes y spillovers, se diseñan dos redes hipotéticas y se ilustran mediante grafos. Estos se construyen bajo los siguientes supuestos. 
Suponga tres regiones, una de primer nivel de desarrollo, intensiva en capital, conocimientos y mercados (en la red se representa mediante un círculo). La segunda región (que se denota con un cuadrado) tiene conocimientos y mercados más que capital. Finalmente, la de tercer nivel (representada con un triángulo) tiene recursos naturales y mercados, pero presenta escasez de conocimientos y de capital.

Cada región tiene un SRI compuesto por: tres clústeres - el agrícola, el industrial y de servicios-, un subsistema universitario, un conjunto de centros de investigación, un sistema financiero $y$, finalmente, un grupo de empresas de servicios de apoyo a la innovación; en suma, siete subsistemas.

También suponga que internamente los subsistemas del SRI están bien conectados; por lo que el conocimiento, los recursos y mercados faltantes los buscan en el extranjero. Finalmente, suponga que la región de primer nivel establece relaciones con la de segundo nivel para buscar mercados y conocimientos. Mientras que con la de tercer nivel establece relaciones para explotar recursos y mercados.

Bajo estos supuestos se construyen los dos grafos. En el primero se representa un PIT entre las regiones de primer y segundo nivel de desarrollo (figura 1).

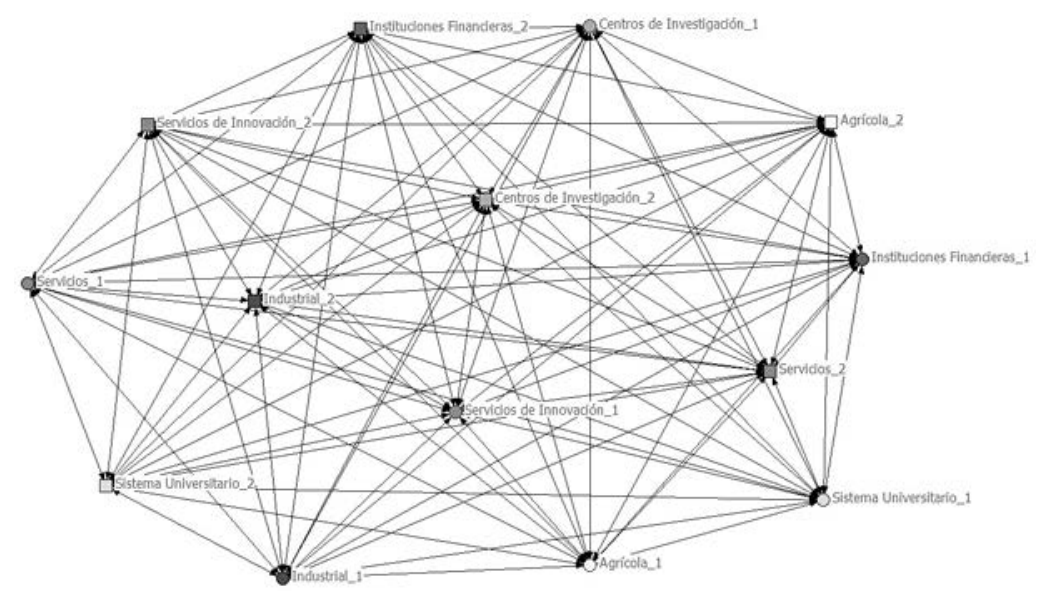

Figura 1. PIT entre sistemas regionales de innovación de primer y segundo nivel de desarrollo

Fuente: elaboración propia. 
Como se observa, los vínculos son de doble vía: se captura el hecho de que como las dos regiones son intensivas en conocimiento, los spillovers tienden a ser recíprocos; por lo que la transferencia de tecnología es del tipo PIT'NL. En términos del ARS, la red es compacta, con un grafo completo.

El segundo grafo describe el PIT entre las regiones de primer y tercer nivel de desarrollo (figura 2).

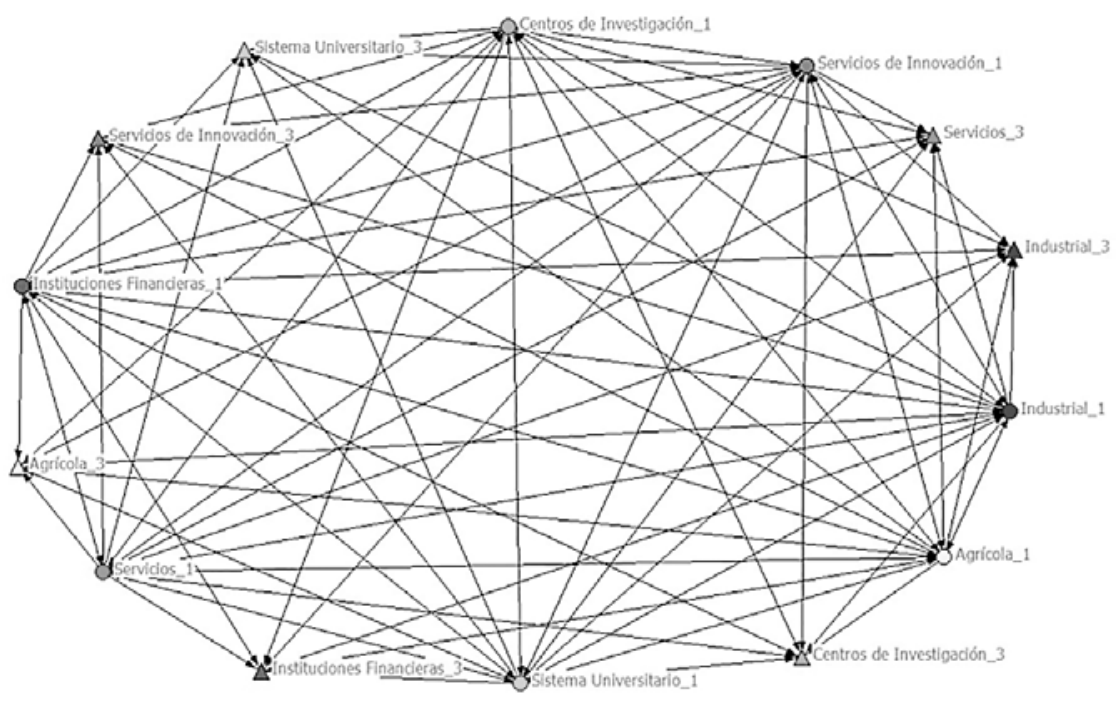

Figura 2. PIT entre sistemas regionales de innovación de primer y tercer nivel de desarrollo

Fuente: elaboración propia.

Dado que la región más desarrollada es intensiva en conocimiento, mientras que la menos desarrollada es intensiva en recursos naturales, los spillovers tienden a ser unilaterales y en una sola vía; por lo que la transferencia de tecnología es del tipo PIT'L. La red es fragmentada y con agujeros estructurales, con un dígrafo o grafo dividido.

La comparación de las propiedades de conectividad y distancia de las dos redes hipotéticas permite deducir las diferencias en el proceso de transferencia de tecnología. Los datos se recogen en la tabla 1. 


\section{Tabla 1}

Indicadores de las propiedades de las redes hipotéticas

\begin{tabular}{lcc}
\hline & RED 1 & RED 2 \\
\hline Tamaño & 182 & 182 \\
Density & 1 & 0,5 \\
No. of Ties & 182 & 91 \\
Std Dev & 0 & 0,5 \\
Avg Degree & 13 & 6,5 \\
Alpha & 1 & 0,933 \\
Average (distancia geodésica) & 1 & 1 \\
Std Dev (distancia) & 0 & 0 \\
Distance-based cohesion (“Compactness”) & 1 & 0,5 \\
Distance-weighted fragmentation (“Breadth”) & 0 & 0,5 \\
Freq distancias & 1 & 182 \\
& Arco: $100 \%$ & Arco: $46,2 \%$ \\
Reciprocidad & Díada: $100 \%$ & Díada: $30,0 \%$ \\
Transitividad & $100,00 \%$ & $23,08 \%$ \\
\hline
\end{tabular}

Fuente: elaboración propia.

Aquí la red 1 representa al PIT'NL y la red 2, al PIT'L. Siguiendo a Hanneman (2000), la conectividad se evalúa mediante los indicadores de tamaño, densidad, grado nodal y alpha. La distancia se mide mediante la distancia geodésica y el diámetro. Finalmente, el equilibrio se analiza con base en los parámetros de transitividad y reciprocidad.

Los datos advierten que entre el PIT'NL y el PIT'L existen diferencias de funcionamiento. El proceso involucra 14 agentes (7 por cada región), el tamaño de la red que describe ambos procesos es similar, 182 enlaces posibles. Pero la densidad que mide las interacciones o relaciones reales en el PIT'NL es $100 \%$ (con una DS=0), mientras en la red 2 es de $0,5 \%$ (con DS=0,5) apenas la mitad. En cuanto al grado nodal, que evalúa el número de agentes a los que algún otro puede acceder, en la red 1, es 13 y en la red 2, 6,5; lo que indica que en el PIT'NL un agente puede acceder a todos los demás; mientras que en el PIT'L solo a la mitad. En cuanto al índice alpha, que mide el nivel de conectividad de la red, en el PIT'NL es 1 y en el PIT'L, 0,933.

Ahora bien, en cuanto a la propiedad de distancia, que indica el esfuerzo o el número de pasos que un agente debe dar para contactar con otro, también existen diferencias 
entre el PIT'NL y el PIT'L. En la tabla 1 se muestra que la distancia geodésica promedio es 1 en ambas redes, con desviación estándar de 0 ; pero la distancia de cohesión y fragmentación en la red 1 es 1 y 0 , respectivamente, mientras que en la red 2 es 0,5 y 0,5 . Lo que indica que en el PIT'NL los agentes realizan menos esfuerzos para entrar en contacto con otros.

La propiedad de equilibrio o linealidad de la red se evalúa a través de la reciprocidad, que mide si los contactos entre dos agentes van en doble vía; y la transitividad, que indica si existe reciprocidad entre tres agentes. Aquí también los datos reflejan divergencias en el proceso de transferencia. Se muestra que en la red 1, la reciprocidad díada es $100 \%$, mientras que en la red 2 es $30 \%$. La transitividad es de $100 \%$ en la red 1 y de $23,08 \%$ en la red 2. De aquí se deduce que el PIT'NL es un proceso recíproco y no lineal y equilibrado, dado que es altamente probable que, si un agente $A$ intercambia conocimiento con B y este con C, A también lo hará con C. Por su parte, el PIT'L, al tener menos reciprocidad y transitividad, será un proceso lineal y desequilibrado, porque si un agente $A$ intercambia con $B$, no es seguro que $B$ le responda, $y$ mucho menos que entre en contacto con otro agente $C$.

Llegados a este punto del análisis, se está en condiciones de proponer las hipótesis que guían esta investigación.

H1: Dado un conjunto de regiones con capacidad para transferir tecnología, aquí se plantea que el PIT entre las regiones más desarrolladas experimenta mayor conectividad, menor distancia, mayor equilibrio o reciprocidad, y mayor interactividad o no linealidad que el PIT entre regiones de diferente nivel de desarrollo.

H2: Una región será más transmisora que receptora de tecnología, entre más alta sea la posición que ocupa en la globalización, la economía del conocimiento y competitividad; y será más receptora que transmisora, entre menor sea el grado de globalización, nivel de conocimiento y competitividad.

\section{Metodología}

Para probar las hipótesis de trabajo, aquí se sigue una metodología en tres pasos, en primer lugar, se identifican las regiones objeto de análisis; en segundo término, se evalúa el rol de las regiones como transmisoras y/o receptoras de tecnología; luego se construyen las redes que describen los diferentes tipos de PIT. 


\section{Identificación de las regiones}

Los territorios objeto de análisis son los de segundo nivel de toma de decisiones administrativas de los países, calificados como TL2 por la OCDE o NUTS2 por la Unión Europea, y sus equivalentes en Latinoamérica. Para evaluar las regiones más competitivas, se tomaron los rankings: WKCI del 2014, que identificó a 145 regiones como más competitivas en conocimiento; el GaWC de 2016, que calificó 298 unidades territoriales como globales. Por su parte, el estudio de la OCDE (2012) Promoviendo el crecimiento en todas las regiones, evaluó 23 ciudades como innovadoras. El libro de la CEPAL titulado Economía y territorio en América Latina y el Caribe (Ramírez, Silva y Cuervo, 2009), identifica a 32 territorios como ganadores. Y el estudio Los sistemas regionales de innovación en América Latina (Llisterri, Pietrobelli y Larsson, 2011) del BID, identificó a 9 regiones con buenos sistemas de innovación.

Se seleccionaron las regiones que calificaran en al menos tres de los cinco rankings. Para ello se construyó una matriz de coincidencias. Los resultados se recogen en la tabla 2.

\section{Tabla 2}

Muestra de las principales RGBC en el mundo con base en las TL2 de la OCDE

\begin{tabular}{ccccc}
\hline $\begin{array}{c}\text { Fuentes de } \\
\text { información }\end{array}$ & $\begin{array}{c}\text { Regiones ganadoras } \\
\text { identificadas }\end{array}$ & $\begin{array}{c}\text { Regiones } \\
\text { clasificadas }\end{array}$ & $\begin{array}{c}\text { Resultado de la matriz } \\
\text { de coincidencias }\end{array}$ & $\begin{array}{c}\text { Número de regiones } \\
\text { que coinciden }\end{array}$ \\
\hline WKCI & 145 & 108 & WKCI/GaWC/OCDE & 108 \\
GaWC & 298 & 116 & & \\
OCDE & 23 & 23 & & \\
CEPAL & 182 & 32 & BID/CEPAL/OCDE & 32 \\
BID & 9 & 9 & & 140 \\
Total & 514 & 288 & Total & \\
\hline
\end{tabular}

Nota: de las regiones clasificadas en WKCI, GaWC y OCDE, se incorporan 108 en el presente estudio; mientras que, de la OCDE, CEPAL y el BID se incorporan 32 regiones, principalmente las latinoamericanas.

Fuente: elaboración propia.

La tabla 2 muestra que 140 territorios califican como objeto de análisis, de los cuales 108 coinciden en los rankings del WKCI, el GaWC y la OCDE, y 32 aparecen en los rankings de la OCDE, BID, GaWC y la CEPAL.

El paso siguiente fue clasificarlos de mayor a menor nivel de competitividad. Para ello, se tomó como variable discriminante el nivel de PIB per cápita y su localización en el 
nivel de competitividad. Se definieron como menos competitivas o regiones del grupo 3 (RG3), los territorios de renta media y media alta de entre $3150 \$$ hasta $25000 \$$ y que califican según el Banco Mundial como territorios que basan su competitividad en recursos naturales o requerimientos básicos. Por su parte, son regiones del grupo 2 (RG2) en competitividad, las regiones con un nivel de renta comprendido entre los $25000 \$$ hasta los $36000 \$$ y que se identifican como regiones basadas en la eficiencia de los factores. Finalmente, se definen como regiones del grupo 1 (RG1) en competitividad, las que tienen más de $36000 \$$ de ingreso per cápita y que fundamentan su competitividad en la innovación.

Una vez aplicados estos criterios, se llegó a una muestra de 137 regiones, donde clasificaron 56 en el grupo 1, 48 en el 2, y 33 en el tres.

\section{Identificación de las regiones como receptoras o transmisoras de tecnología}

Para identificar el rol de las regiones se aplicó la técnica de análisis de correspondencia múltiple (ACM), técnica muy útil cuando se requiere determinar los atributos de un sujeto (Greenacre, 2008). Con ese fin se tomaron las categorías en que los rankings de KWCI, GaWC y el Banco Mundial, clasifican a las regiones. Los cálculos del ACM se recogen en la tabla 3.

\section{Tabla 3}

Análisis de dependencia entre los rankings de clasificación regional

\begin{tabular}{|c|c|c|c|}
\hline & \multirow[b]{2}{*}{ Dependencia estadística } & Variabilidad explicada & \multirow{2}{*}{$\begin{array}{c}\text { Correlación } \\
(\%)\end{array}$} \\
\hline & & $\begin{array}{c}\text { Dos componentes } \\
(\%)\end{array}$ & \\
\hline GaWC/KWCI & Existe $(p$-valor $=0,066)$ & 91 & 22,1 \\
\hline GaWC/Renta & Existe $(p$-valor $=0,0458)$ & 100 & 27,5 \\
\hline KWCI/Renta & Existe $(p$-valor $=0)$ & 100 & 66,3 \\
\hline
\end{tabular}

Fuente: elaboración propia a partir de resultados del programa R.

El cálculo del ACM arroja que existe relación positiva y dependencia estadística entre las tres variables. Lo que significa que una región, en mayor o menor medida, es a la vez globalizada bajo los atributos del GaWC, competitiva en la economía del conocimiento del KWCI y competitiva según el Banco Mundial. 
Verificada la correspondencia de atributos, se clasificaron las regiones en seis clases en función de su rol como transmisoras o receptoras, según los siguientes criterios: se consideran como regiones de clase 1 aquellas más transmisoras que receptoras de tecnología, estos son aquellos territorios que basan su competitividad en la innovación, son líderes, seguidores o emergentes, en la economía del conocimiento, en relación directa con la posición que ocupen en el grado de globalización; por lo que son más transmisoras las regiones alpha, dado que enlazan a todo el mundo, que las suficientes, que solo enlazan a la región con los países geográficamente más cercanos.

Por su parte, son de clase 6 las regiones más receptoras que transmisoras de tecnología, los territorios más intensivos en recursos naturales, los que califican como de transición o rezagadas en la economía del conocimiento, y como gamma o suficientes en globalización; porque son muy poco intensivos en conocimientos y tienen bajos enlaces con el resto del mundo.

A partir de una tabla disyuntiva que arroja el ACM, se clasificaron las 137 regiones en 6 clases, según su rol como transmisoras y/o receptoras de tecnología dado el grupo en competitividad. Esta estructura se muestra en la tabla 4.

\section{Tabla 4}

Clasificación de las regiones como receptoras o transmisoras de tecnología

\begin{tabular}{|c|c|c|c|c|}
\hline Clase & Grupo & KWCI & GaWC & Total regiones \\
\hline 1 & 1 & $\begin{array}{l}\text { Líderes, o seguidoras, o } \\
\text { emergentes }\end{array}$ & alph (16), beta (8), gamma (9), sufic (7), insufic (0) & 40 \\
\hline 2 & 1 & Transición & $\operatorname{alph}(8)$, beta (3), gamma (0), sufic (3), insufic (2) & 16 \\
\hline 3 & 2 & $\begin{array}{l}\text { Líderes, o seguidoras, o } \\
\text { emergentes }\end{array}$ & $\operatorname{alph}(4)$, beta (3), gamma (3), sufic (4), insufic (9) & 23 \\
\hline 4 & 2 & Transición o rezagadas & alph (6), beta (5), gamma (6), sufic (5), insufic (3) & 25 \\
\hline 5 & 3 & Emergentes & $\operatorname{alph}(3)$, beta (1), gamma (3), sufic (2), insufic (0) & 9 \\
\hline 6 & 3 & Transición o rezagadas & $\operatorname{alph}(3)$, beta (3), gamma (3), sufic (7), insufic (8) & 24 \\
\hline
\end{tabular}

Fuente: elaboración propia.

Calificaron como del grupo 1, o más innovadoras, 56 regiones; de las cuales 40 son de clase 1 y 16 de clase 2. Del grupo 2, o las basadas en la eficiencia de los factores, clasificaron 48; de las cuales son de clase 3, 23 y clase 4, 25 regiones. Por otra parte, del grupo 3, o intensivas en recursos naturales, se contabilizaron 33 regiones, de las cuales 9 son de clase 5 , mientras que las 24 regiones restantes son de clase 6 . 


\section{Determinación de los vínculos de transferencia de tecnología}

Una vez definido el rol de las regiones, el paso siguiente fue establecer los vínculos 0 enlaces entre ellas. De nuevo la técnica del ACM fue de gran utilidad porque permite obtener el perfil fila o columna de una determinada variable (Greenacre, 2008). Aquí se calculó la probabilidad de que, si una región ocupa un lugar en la economía del conocimiento según el KWCI, puede que ocupe un determinado lugar en globalización en el GaWC. En términos de transferencia de tecnología mediante la probabilidad condicionada, se puede inferir que dado el nivel de conocimiento que posee una región, cual es la factibilidad de globalizarse, intercambiando tecnología y conocimiento, mediante la inversión extranjera, pasajeros e información.

Para establecer la probabilidad de los vínculos, se siguieron dos pasos: en primer lugar, se calculó el perfil fila de cada una de las categorías de las regiones; es decir, dada la intensidad en conocimiento de una región, como líder, seguidora, emergente, en transición o rezagada, se calculó que fuera alpha, beta, gamma o suficiente en globalización. El segundo paso fue calcular la probabilidad de que ocurra el evento de transferencia de tecnología entre las regiones. Para ello se multiplicó el perfil fila de las regiones clase $X$ con cada uno de los perfiles columna de las demás clases. Cada multiplicación arrojó un único valor, que determina la probabilidad de transferencia de conocimiento entre las distintas clases de regiones. Estos resultados se recogen en la tabla 5.

\section{Tabla 5}

Matriz de probabilidades de vínculos entre regiones

\begin{tabular}{ccccccc}
\hline & Clase 1 & Clase 2 & Clase 3 & Clase 4 & Clase 5 & Clase 6 \\
\hline Clase 1 & 0,281250 & & & & & \\
Clase 2 & 0,270312 & 0,335937 & & & & \\
Clase 3 & 0,155434 & 0,192935 & 0,2476371 & & & \\
Clase 4 & 0,225 & 0,21 & 0,1808696 & 0,209600 & & \\
Clase 5 & 0,269444 & 0,229167 & 0,1545894 & 0,226666 & 0,283951 & \\
Clase 6 & 0,154166 & 0,182292 & 0,2355072 & 0,183333 & 0,162037 & 0,243055 \\
\hline
\end{tabular}

Fuente: elaboración propia.

La tabla muestra la probabilidad de que existan vínculos entre las regiones. Se destaca que las mayores probabilidades están en los enlaces entre las regiones de la misma clase. Las regiones clase 1 se relacionan bien con las regiones de las otras clases, especialmente con clase 2 y clase 5 , y se relacionan muy poco con clase 3 . A partir de esta tabla 
se definen como vínculos fuertes y de doble vía los que superan 0,25 y de una sola vía, las menores de este valor.

\section{Construcción de las redes de transferencia de tecnología entre regiones}

Para la construcción de las redes objeto de análisis, se procedió de la siguiente manera: en primer lugar, las regiones se clasificaron en tres grupos, las del grupo 1 se denotan mediante círculos, las del 2 con cuadrados y las del 3 con triángulos. Por otra parte, a cada una de las clases de regiones se les asignó un color, así: las de clase 1 son de color rojo, las de la 2 son naranjas, las de la 3 son amarillas, las de la 4 son verdes, las de 5 moradas y las de clase 6 grises.

Dado que se pretenden probar las diferencias en el proceso de transferencia de tecnología entre regiones de diferente nivel de desarrollo y el rol que las regiones juegan como receptoras o transmisoras de tecnología, se diseñaron tres redes: la primera, entre las regiones del grupo 1 y 2, que incluyen a las clases 1, 2, 3 y 4; para lo que se elaboró una matriz de 104 * 104 regiones. Por su parte, en la segunda red se involucraron a las regiones de los grupos 1 y 3, con las clases $1,2,5$ y 6 ; para lo que se diseñó una matriz de $99 * 99$. Finalmente, la red 3 se construyó tomando las regiones de los grupos 2 y 3 , y las clases 3, 4, 5 y 6; aquí la matriz fue de 81 * 81 .

Para establecer los vínculos, en todas las tres redes se partió de los cálculos de la tabla 5. Se planteó que existe una relación de doble vía o bidireccional entre dos regiones si la probabilidad de que ocurra un evento típico de transferencia de tecnología es mayor o igual a 0,25. Mientras que se plantea que existe una relación en una sola vía de una región de clase superior a una inferior si la probabilidad de ocurrencia de la transferencia de tecnología es menor a este valor.

Se usó el programa Ucinet para calcular las propiedades de conectividad, distancia, equilibrio de la red, y los indicadores de cercanía, poder e intermediación, y el programa Net Draw, para obtener los grafos de las redes.

\section{Análisis de resultados: características del PIT entre regiones}

Antes de presentar los resultados, se describen las características de las regiones objeto de análisis, en función de la localización geográfica y posicionamiento en el GaWC y el KWCI. Dentro del grupo de las 137 regiones, 15 de las 40 regiones que componen 
el RG1, se encuentran en Estados Unidos, 3 en Alemania, 2 en Canadá y 1 se localiza en cada uno de los siguientes países: China, Austria, Suecia, Suiza, Noruega, Francia, Australia, República de Singapur, República Eslovaca, República Checa, Reino Unido, Bélgica y Luxemburgo. En cuanto al grado de globalización de las RG1, 8 califican como alpha, 6 alpha+, 10 betas, 3 gammas, 5 suficientes y 1 no-globalizada. Finalmente, según su competitividad en la economía del conocimiento, 9 de las RG1 son líderes, 12 seguidoras, 9 están en transición y 3 son emergentes.

Ahora bien, de las RG2 identificadas, Alemania y Suecia cuentan cada uno con 4; Reino Unido, Finlandia y Japón, con 3; Australia, Canadá y Estados Unidos, con 2, e Israel, Francia, Hungría, Islandia, Brasil, Dinamarca, Italia, Korea del Sur, Austria y Países Bajos cuentan cada uno con 1. En función del grado de globalización, 1 es alpha, 1 más es alpha+, 9 son beta, 3 gammas, 7 suficientes, 12 no-globalizadas. En relación con la economía del conocimiento, de las RG2 solo 1 es líder, 11 seguidoras, 6 emergentes, 11 están en transición y 3 rezagadas.

Por último, en cuanto a su localización, se tiene que 8 de las RG3 se encuentran en México, 6 en Brasil, 5 en China, 4 en Colombia, 2 en India, 2 en Chile y 1 en Argentina, Perú, Letonia, Lituania, Estonia y España. Mientras que, en relación a su grado de globalización, 5 son alpha, 1 es alpha+, 5 son betas, 5 más son gammas, 9 son suficientes y 8 no-globalizadas. En cuanto al nivel de conocimiento, de las RG3, 23 son rezagadas, 9 emergentes, 1 en transición y ninguna es seguidora ni líder. En la tabla 6 se presentan los resultados obtenidos.

\section{Tabla 6}

Indicadores de la dinámica del PIT entre regiones

\begin{tabular}{lccc}
\hline \multicolumn{1}{c}{ Indicadores } & RED 1 (RG1-RG2) & RED 2 (RG1-RG3) & RED 3 (RG2-RG3) \\
\hline Tamaño & 10712 & 7832 & 6480 \\
Density & 0,725 & 0,449 & 0,282 \\
No. of Ties & 7764 & 3517 & 1826 \\
Std Dev & 0,447 & 0,497 & 0,45 \\
Avg Degree & 74,654 & 39,517 & 22,543 \\
Alpha & 0,996 & 0,986 & 0,969 \\
Average (distancia geodésica) & 1,1 & 1,3 & 1,4 \\
Std Dev (distancia) & 0,3 & 0,5 & 0,6 \\
Distance-based cohesion (“Compactness”) & 0,757 & 0,55 & 0,347 \\
Distance-weighted fragmentation (“Breadth”) & 0,243 & 0,45 & 0,653 \\
\hline
\end{tabular}




\begin{tabular}{|c|c|c|c|}
\hline Indicadores & RED 1 (RG1-RG2) & RED 2 (RG1-RG3) & RED 3 (RG2-RG3) \\
\hline Freq distancias & $\begin{array}{rl}1 & 7764 \\
2 & 682 \\
\text { NA } & 2266\end{array}$ & $\begin{array}{cc}1 & 3517 \\
2 & 1576 \\
\text { NA } & 2739\end{array}$ & $\begin{array}{rl}1 & 1826 \\
2 & 759 \\
3 & 135 \\
\text { NA } & 3760\end{array}$ \\
\hline Reciprocidad & $\begin{array}{l}\text { Arco: } 85,5 \% \\
\text { Díada: } 74,7 \%\end{array}$ & $\begin{array}{l}\text { Arco: } 52,6 \% \\
\text { Díada: } 35,7 \%\end{array}$ & $\begin{array}{c}\text { Arco: } 40,5 \% \\
\text { Díada: } 25,4 \%\end{array}$ \\
\hline Transitividad & $53,77 \%$ & $16,38 \%$ & $6,87 \%$ \\
\hline
\end{tabular}

Fuente: elaboración propia.

En esta tabla se recogen tres tipos de indicadores, los que describen la propiedad de la conectividad, que incluye la densidad, el grado nodal y el indicador alpha. El segundo conjunto de indicadores son los de la distancia; aquí entran la distancia geodésica, el diámetro, la compactación, la fragmentación. Por último, se presentan los indicadores del equilibrio y linealidad del PIT, medido mediante la reciprocidad y transitividad de la red. A partir de la tabla 6 , se deducen las diferencias entre los tres PIT.

\section{EI PIT entre las regiones de primer y segundo nivel de desarrollo}

La estructura del PIT entre las regiones de esta clase se recoge en la figura 3. Aquí se describe el PIT entre las regiones más desarrolladas del mundo.

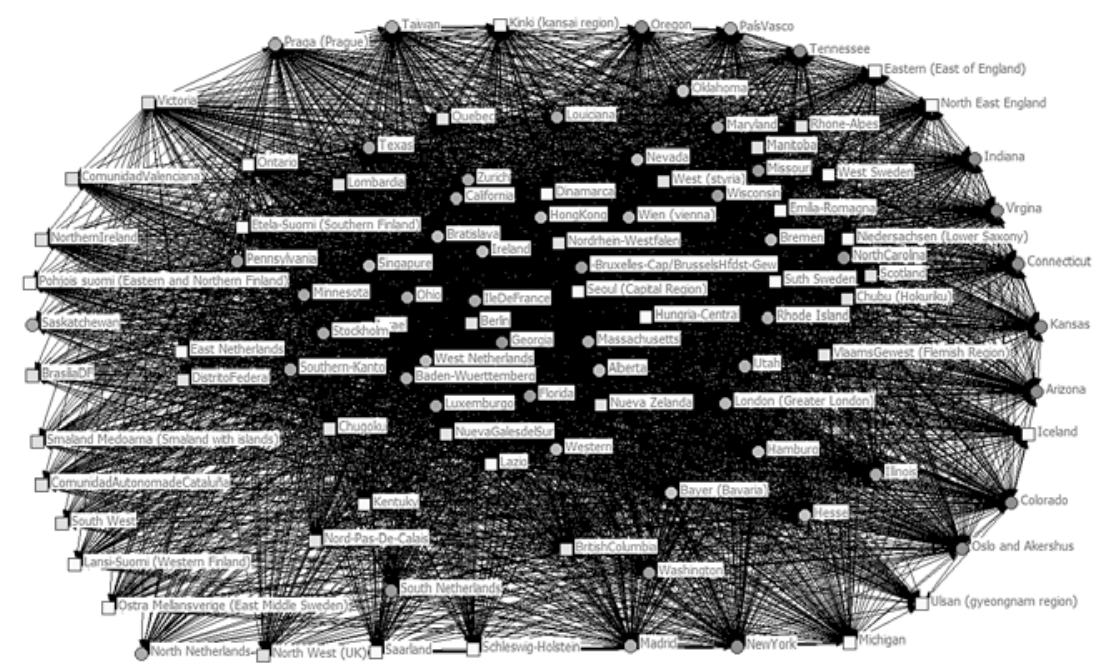

Figura 3. Representación del PIT'NL: entre regiones de primer y segundo nivel de desarrollo Fuente: elaboración propia. 
El grafo de la figura 3 es muy similar al de la figura 1, con spillovers recíprocos, en donde las relaciones van en doble sentido. Los indicadores de las propiedades de la red permiten explicar el PIT entre esta clase de regiones. Una mirada a la primera columna de la tabla muestra que la red 1 evidencia que el PIT entre las regiones de esta clase es el más complejo y de mayor tamaño; debido a que como participan 104 regiones, tiene un tamaño de 10712 relaciones posibles. Por su parte, la densidad del $72 \%$ indica que se presentan 7764 de las relaciones reales. En esta red se percibe que en el PIT de este tipo cada región tiene intercambios con el $75 \%$ de las regiones, en promedio $(74,654$ según datos del degree). La conectividad alpha es de 0,996, muy cercana a 1, lo que indica que es un PIT muy conectado. De aquí se infiere que el PIT entre las regiones más desarrolladas del mundo tiende a ser de muy pocos agujeros estructurales, por lo que el PIT es un proceso fluido y con pocas barreras o agentes que lo frenen.

Los resultados aquí obtenidos justifican la afirmación de Carayannis et ál. (2017) respecto a que los clústeres localizados en las regiones de Estado Unidos, Europa Occidental y Asia están altamente conectados. Y que Narula y Pineli (2017) proponen que en estos países la IED fluye más rápido gracias a la homogeneidad de las instituciones y de la estructura económica. Usando el ARS también se prueba que las regiones más intensivas en el PIT son las calificadas por la OCDE (2012), el GaWC (2016) y el KWCI (2014) como las más innovadoras, alpha o beta en globalización y líderes o seguidoras en la economía del conocimiento.

Los resultados de la tabla 6 permiten explicar por qué el PIT entre regiones desarrolladas es un PIT'NL; esto lo certifica el hecho de que la distancia geodésica o el camino más corto entre un par de regiones es de 1,1, con una desviación estándar de 0,3. En este PIT, el 72,5\% de las relaciones están a un paso (7764 relaciones con una distancia de 1). Así mismo, el $6,4 \%$ (equivalentes a 682 con distancia de 2 ) y el $21 \mathrm{v}, 2 \%$ de las relaciones son de distancia infinita (2266) o no tienen caminos ciertos para vincularse. Esta red experimenta una cohesión de 0,757 y de fragmentación de 0,243; de aquí se deduce que es un PIT muy articulado. La proximidad entre las regiones que participan en el PIT'NL se presenta gracias a la reducción en los costos de transporte y comunicación; lo que ha reducido la distancia geográfica; además, la globalización y la presencia de organismos multilaterales como la OCDE, la UE y la OMC también han homogeneizado las políticas y las instituciones, lo que ha aumentado la proximidad institucionaly cognitiva (Balland, Boschma y Frenken, 2015).

Por otro lado, los datos de la red evidencian que la no linealidad del PIT se debe a que la red experimenta una reciprocidad equivalente a $74,7 \%$. Y una transitividad de $53,77 \%$. 
Lo que indica que, si una región A establece un PIT con otra B y a su vez B contacta con C, entonces es altamente probable que A contacte con C. Al respecto Balland et ál. (2015) han mostrado que, entre mayor cercanía entre regiones, mayor será la probabilidad de transferencia de conocimiento; lo que también han corroborado Gertler y Levitte (2003).

La transferencia de tecnología entre regiones desarrolladas y de menor nivel de desarrollo

Debido a que la hipótesis de este trabajo busca establecer las diferencias en el PIT entre regiones de diferente nivel de desarrollo, en este apartado se evalúan dos PIT, el de las regiones de primer y tercer nivel, y el de las de segundo y tercer nivel de desarrollo; es decir se evalúa el PIT entre regiones de distinta estructura económica.

\section{EL PIT ENTRE LAS REGIONES DE PRIMER Y TERCER NIVEL DE DESARROLLO}

Los rasgos del PIT entre estas regiones se recogen en la red 2 y en la figura 4.

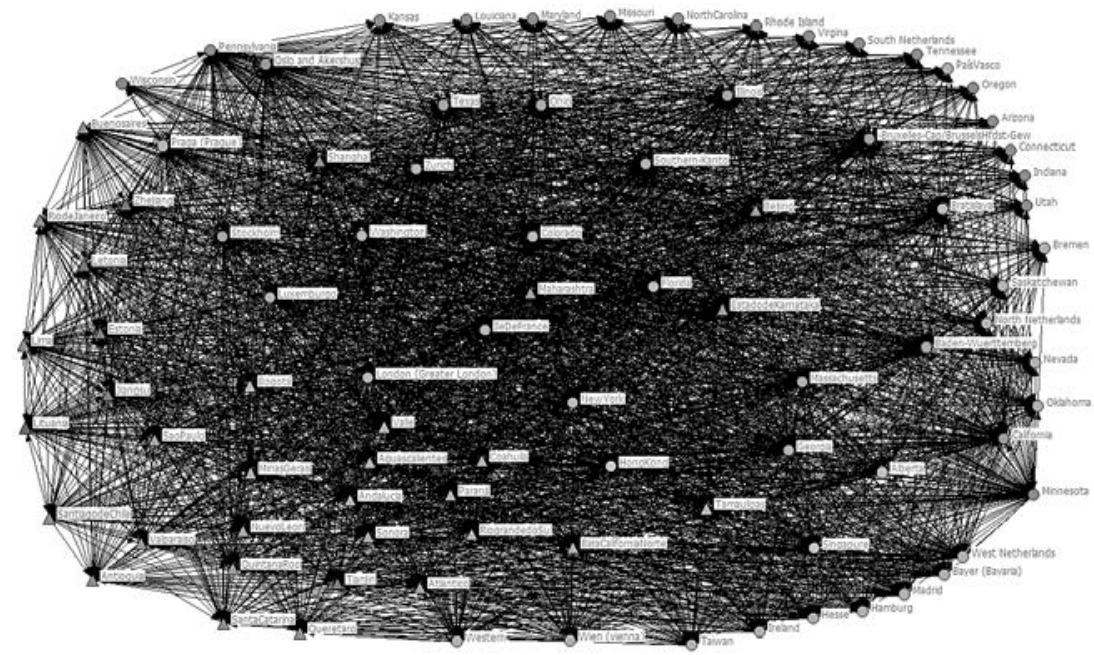

Figura 4. Representación del PIT'L: entre regiones de primer y tercer nivel de desarrollo

Fuente: elaboración propia.

El gráfico de la figura 4 es semejante al de la figura 2; aquí se evidencia que entre las regiones consultadas se configura un PIT donde predominan los vínculos que van en una sola dirección, esto es, una red con spillovers unilaterales. Esta es la forma tradicional en la que se 
transfiere tecnología a las regiones y los países en vía de desarrollo (Narula, 2010); se afirma que este es un proceso lineal porque el conocimiento circula en un solo sentido. Las características del PIT'L se describen con los indicadores de la columna 2 de la tabla 6.

Aquí se observa que el PIT entre las regiones de primer y tercer nivel de desarrollo involucra a 99 regiones, por lo que el tamaño es de 7832 posibles conexiones; no obstante, la densidad es de 3517 vínculos reales (con una DS de 0,497), equivalentes al $44,9 \%$ del total. En esta red cada región tiene la posibilidad de hacer intercambios, en promedio, con el 45\% de las regiones; debido a que el índice de degree es de 39,517 enlaces. Por su parte, el índice de conectividad es del 0,98 ; lo que indica que la red tiene un nivel de conexión aceptable. Estos datos evidencian que esta red es de una estructura frágil, con muchos agujeros estructurales, con tendencia a formar subredes, con un PIT jerárquico y concentrado en pocas regiones.

Los indicadores de las propiedades de esta red permiten comprender por qué la transferencia de tecnología hacia los países en desarrollo es menos intensa, más escasa y específica, basada más en tecnologías duras o máquinas (Albornoz y López, 2015). Narula y Michel (2009) afirman que la IED entre los países en desarrollo es muy poca, debido a que las multinacionales tienen solo incentivos para extraer riqueza y no para coproducir conocimiento. Es de resaltar que las regiones que participan en este PIT tienden a fundamentar la competitividad en recursos naturales (OCDE, 2012); califican como gamma y suficientes en el ranking del GaWC (2016), y como de transición y rezagadas en la economía del conocimiento Huggins et ál. (2014); lo cual indica que estas regiones son más receptoras que transmisoras de tecnología.

Ahora bien, en cuanto la propiedad de distancia de la red, se evidencia que la distancia geodésica entre las regiones RG1 y RG3 en promedio es de 1,3 (con una DS=0,5); aquí, el 44,9\% (3517) de las relaciones están a un paso de establecer contactos, mientras que el $20,1 \%$ (1576) lo están a 2 pasos; y el 35\% (2739) de relaciones no pueden articularse. Lo cual justifica que el nivel de fragmentación sea de 0,45 y el de compactación de 0,55. Aunque los costos de transporte y comunicación han disminuido la distancia entre agentes de las regiones, todavía persisten las brechas tecnológicas e institucionales entre ellos (Narula y Pineli, 2017), lo que no ha permitido una mayor proximidad cognitiva ni la organizacional. En este sentido, Casas y Gortari (2001), y Caicedo (2012) han mostrado que la relación entre las organizaciones de los SRI en México y Colombia experimentan una baja proximidad y alta fragmentación; por lo tanto, existe una baja probabilidad de trasferencia de conocimiento. 
En cuanto a la linealidad del PIT entre estas regiones, los indicadores de reciprocidad y transitividad arrojan luces. En la tabla se observa que la reciprocidad entre las regiones de primer y tercer nivel de desarrollo es muy baja, apenas del 35,7\%, inferior al $50 \%$. Por otra parte, las relaciones entre tres o más agentes es mucho más crítica, apenas si alcanza el 16,38\%; por lo que se infiere que el proceso de transferencia entre este tipo de regiones es más de un $\mathrm{PIT}^{\prime} \mathrm{L}$, direccionado unilateralmente.

En este tipo de PIT las empresas locales se enganchan débilmente a las cadenas globales de valor y, cuando participan, lo hacen como proveedores de materias primas, compitiendo con proveedores de otras partes del mundo, con escasa rentabilidad de sus productos (Pietrobelli y Rabelloti, 2011).

\section{LA TRANSFERENCIA DE TECNOLOGÍA ENTRE LAS REGIONES DE SEGUNDO Y TERCER NIVEL DE DESARROLLO}

En esta investigación se consideró pertinente investigar el PIT entre regiones de segundo y tercer nivel de desarrollo. Las particularidades de este tipo de PIT se recogen en la red 3 y se ilustran en la figura 5 .

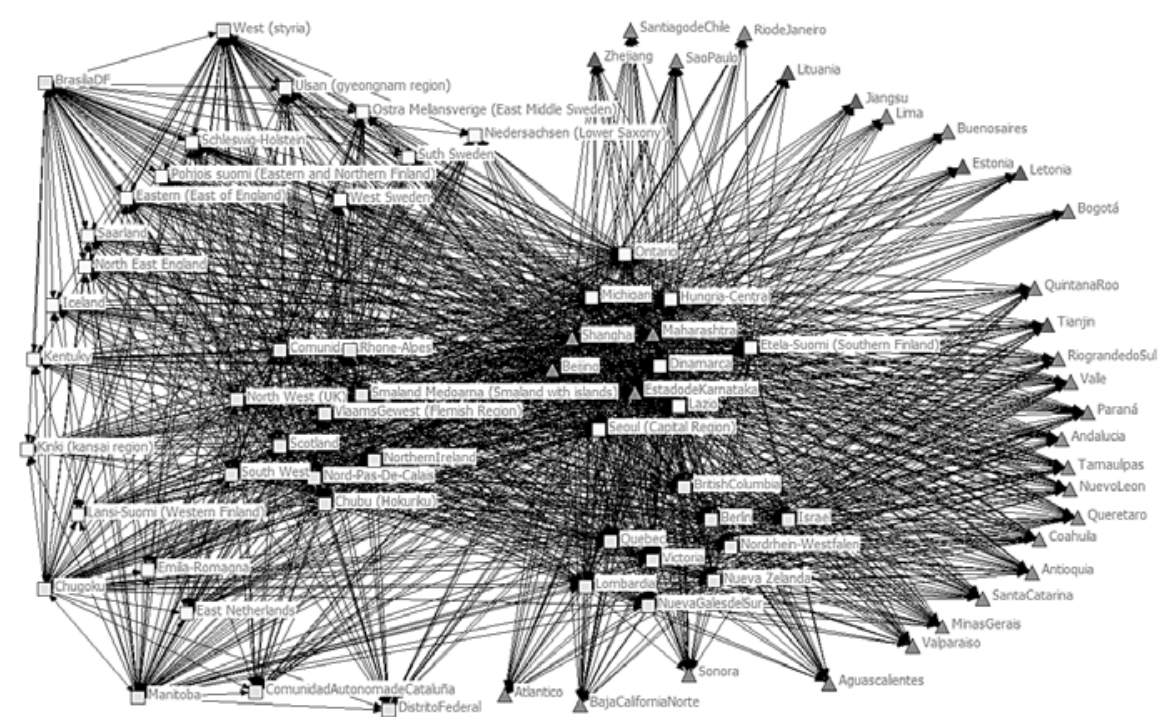

Figura 5. Representación del PIT'L: entre regiones de segundo y tercer nivel de desarrollo Fuente: elaboración propia. 
En el grafo de la figura 5 se evidencia que el PIT entre estas regiones es similar al de la figura 2; lo que permite deducir que este proceso es más del tipo PIT'L. Ahora bien, como se muestra en la columna 3 de la tabla 6 , intervienen 81 regiones; el tamaño de esta red es de 6480 relaciones posibles. Se observa una densidad de $28,2 \%$, dado que 1826 vínculos son reales (con una D'S de 0,45 ). En esta red una región se relaciona como máximo en promedio con 23 regiones. El PIT tiene una conectividad alpha de 0,969. De aquí se infiere que este PIT es el más débil, con menor tamaño, muchos agujeros estructurales y una menor diversificación entre las alternativas de transferencia.

Los datos permiten inferir que en este PIT'L las redes tienden a ser más simples, con vínculos en una sola vía, muy abiertas y con muchos agujeros que provocan fugas de información y conocimiento (Casas et ál., 2013; Caicedo, 2012).

Los resultados son los esperados, debido fundamentalmente a que aquí participan regiones de segundo nivel que buscan intercambiar con otras de menor desarrollo que apenas logran ser de transición o rezagadas en la economía del conocimiento, y gamma, suficientes y las menos globalizadas. Por consiguiente, la IED se localiza en menor proporción, debido a que los SRI de estas regiones tienden a ser débiles, fragmentados y desarticulados (Caicedo, 2012).

En términos prácticos del PIT'L, los spillovers que les quedan a las regiones de menor desarrollo son formas de organización administrativas, proveedores formados alrededor de unas tecnologías específicas, instalaciones y algunas infraestructuras. Spillovers que en muchos casos no incrementan la PTF y que, contrario a lo esperado, profundizan las desigualdades sociales (Suanes y Roca-Sagalés, 2015).

El proceso experimenta rezagos en el flujo de la IED, a la vez que en la colaboración de las relaciones para la creación y explotación del conocimiento y la cooperación.

Ahora bien, esta red experimenta una mayor disfuncionalidad que la anterior; como se observa, la distancia geodésica es de 1,4 con una desviación de 0,6; con un diámetro de 3 . Aquí existe un 28,2\% (1826) de relaciones en las que una región solo necesita un paso para intercambiar con otra, un 11,7\% (759) necesitan 2 pasos y un 2,1\% (135) necesitan 3; pero más del $50 \%$ de las relaciones no tienen salida o no se cumplen, dado que se evidencia que 3760 tienen distancia infinita. En cuanto a la cohesión, esta es de 0,347 y la fragmentación, de 0,653. Todo lo anterior evidencia que entre estas clases de regiones el PIT es muy débil, poco fluido y con la mayor cantidad de circuitos rotos. 
En este sentido, el PIT entre estas regiones es más lineal y simple que el de la anterior red. Esto lo refleja el hecho de que la reciprocidad de díada es de $25,4 \%$ y la transitividad de $6,87 \%$. La debilidad de la red se justifica en función de la existencia de relaciones de menor intensidad, al parecer las RG2 y las RG3 prefieren intercambiar tecnología con las RG1 antes que entre ellas.

Aquí, al igual que en la red 2, los vínculos son unidireccionales, en el sentido de que los spillovers y las demás formas de transferencia van de las regiones de segundo a las de tercer nivel de desarrollo.

Para finalizar este apartado de resultados, se puede deducir que se cumple la hipótesis de diferencia entre los PIT de las regiones asociadas a su nivel de desarrollo, debido a que el PIT entre regiones de primer y segundo nivel es mucho más intenso, que el de las de primer y tercero; y segundo y tercer nivel de desarrollo.

\section{Evaluación de las regiones como transmisoras y/o receptoras de tecnología}

El análisis del rol de las regiones como transmisoras y/o receptoras de tecnología parte del reconocimiento de que las regiones no son autosuficientes a la hora de generar toda la tecnología necesaria para competir; por lo que tienen que importar y exportar conocimientos. En esa perspectiva, los índices de closeness, degree y el de intermediación, proporcionan información relevante. Esta se presenta en la tabla 7, donde se recogen los índices de medida del rol de las 6 clases de regiones que se obtuvieron mediante el ACM.

\section{Tabla 7}

Evaluación del rol de las regiones como transmisoras $y /$ o receptoras de tecnología

\begin{tabular}{ccccccc}
\hline Clases & \#Regiones & inCloseness & outCloseness & InDegree & OutDegree & Betweenness \\
\hline \multirow{2}{*}{ Clase 1 } & 4 & 4,348 & 100 & 81 & 103 & 13,373 \\
& 3 & 4,348 & 82,4 & 81 & 81 & 0 \\
Clase 2 & 3 & 4,348 & 100 & 81 & 103 & 13,373 \\
& 3 & 4,348 & 82,4 & 81 & 81 & 0 \\
Clase 3 & 3 & 4,348 & 100 & 81 & 103 & 13,373 \\
& 3 & 2,123 & 1,235 & 81 & 81 & 0 \\
\hline
\end{tabular}




\begin{tabular}{ccccccc}
\hline Clases & \#Regiones & inCloseness & outCloseness & InDegree & OutDegree & Betweenness \\
\hline Clase 4 & 3 & 4,348 & 100 & 81 & 103 & 13,373 \\
& 3 & 4,484 & 0,962 & 51 & 0 & 0 \\
Clase 5 & 4 & 2,066 & 100 & 10 & 80 & 0 \\
Clase 6 & 4 & 4,314 & 1,124 & 50 & 0 & 0 \\
\hline
\end{tabular}

Fuente: elaboración propia.

Como se esperaba, las regiones de clase 1 son las que experimentan mayores valores en outcloseness y outdegree que en inclosenesss e indegree; y tienen los mayores valores de intermediación o betweenness. Estos datos corroboran la idea de que las regiones de esta clase juegan más un rol de transmisoras que de receptoras de tecnología, y resaltan el importante papel que juegan como intermediarias. Aquí se destacan cuatro regiones que poseen el máximo índice de outcloseness, de 100\%; y el mínimo de incloseness, equivalente al 4\% (4,348). A su vez, son regiones que tienen el máximo outdegree (103), un indegree de 81 y un betweenness o intermediación de 13,373. Estas regiones son: Southern-Kanto (Tokio), IleDeFrance, Londres (Greater London) y Nueva York, las cuales son las regiones más competitivas de la clase 1 de las regiones más ricas e innovadoras.

Es de resaltar que, aunque hacen parte del grupo 1, se distinguen dos tipos de regiones de clase 2, uno en el que sobresalen Singapur, Madrid y Taiwán, y otro donde juegan un papel de transmisoras de menor intensidad Oklahoma, Nevada y Bremen por ser del grupo de las regiones más ricas. No obstante, respecto a esta clasificación debe tenerse en cuenta que son regiones alpha, líderes en la economía del conocimiento y fundamentadas en la innovación.

En cuanto a las regiones del grupo 2, sobresale el hecho de que las de clase 3 destacan con índices similares a las del primer tipo de clase 2, en donde las regiones se subdividen en dos categorías; en la primera resaltan Dinamarca, Michigan y Seoul como las más transmisoras de tecnología, y como de menor intensidad se identifican a Kinki (Kansai), Eastern (East of England) y Emilia-Romagna. Estas regiones tienen en promedio un incloseness de 2,123 y un indegree de 81; así como un outcloseness de 1,235, un outdegree de 81 y betweenness de 0 .

En las regiones de clase 4, los resultados muestran que resaltan como las más transmisoras Berlín, Israel y Quebec; y, en un segundo lugar, Comunidad Autónoma de Cataluña, Distrito Federal y Brasilia DF, las cuales tienen un incloseness de 4,484 y un mínimo outcloseness $(0,962)$; así mismo, un indegree de 51, un outdegree de 0 y un betweenness 
de 0 . Las regiones de la clase 3 y 4 , por pertenecer al grupo de las de segundo nivel de desarrollo, son más transmisoras que receptoras; pero si se comparan con las más desarrolladas, tienen menos índices de closseness, degree y betweenness. Aquí las regiones son seguidoras y emergentes en el KWCI, corresponden a aquellas que basan su competitividad en la eficiencia de los factores, a la par que son globalizadas.

Por último, el análisis del rol de las regiones de tercer nivel de desarrollo arroja que entre los territorios de clase 5 se destacan: Shanghai, Maharashtra, Estado de Karnataka y Beijing, con el mínimo incloseness, de 2,066; un indegree de 10 y de intermediación 0; mientras que como transmisoras dentro de esta clase tienen un outcloseness de 100 y un outdegree de 80; lo cual evidencia que entre las regiones de menor nivel de desarrollo son las más transmisoras de tecnología.

Finalmente, en las regiones de clase 6 sobresalen Antioquia, Aguascalientes, Río grande do Sul y Valle, como los territorios que tienen máximo incloseness, equivalente a 4,314; mínimo outcloseness, de 1,124; así como un outdegree de 0 , y de intermediación 0; tienen un indegree de 50. Lo cual indica que entre las regiones de menor desarrollo son las más receptoras y menos transmisoras.

De acuerdo con los hallazgos se puede decir que, en general, las regiones que pertenecen al primer grupo de cada clase (desde las de clase 1 a la 4) son las más transmisoras de tecnología y, aunque son receptoras, su rol es el de generar conocimientos para el resto del mundo.

Por su parte, entre las regiones de menor desarrollo sobresalen las de la clase 5 , donde se destacan regiones que, aunque reciben mucha tecnología del resto del mundo, han logrado algún grado de generación de conocimiento, lo que explica su capacidad para ser competitivas. Por último, se tienen regiones que son consideradas como receptoras netas de tecnología, por lo que están en menor ventaja competitiva y apenas ganan por sus ventajas comparativas gracias a los recursos naturales; esto justifica su rezago en conocimiento y su posicionamiento como gamma y suficientes en globalización.

\section{Conclusiones}

En este trabajo se avanzó en una explicación del proceso de transferencia de tecnología entre los Sistemas Regionales de Innovación -SRI- de las regiones mejor ranqueadas como globales, competitivas en la economía del conocimiento y de mayor crecimiento. 
Se pudo mostrar que cuando se toma a los SRI como agentes transmisores y receptores de tecnología, el proceso internacional de transferencia de tecnología -PIT- sale de la estrecha lógica de la inversión extranjera directa -IED- y se recogen otros procesos, como la colaboración de patentes, convenios universitarios, la movilidad de mano de obra, entre otros, que sin duda se acercan más a la realidad de una economía global basada en el conocimiento.

Se establecieron como vínculos o enlaces, la movilidad de pasajeros, el flujo de entrada y salida de IED, la colaboración en generación de patentes; estos fenómenos se capturaron a partir de la evaluación que hacen de cada región los rankings que sirven de parámetro de análisis. Se construyeron tres redes, una entre las regiones de primer y segundo nivel de desarrollo; la segunda entre las de primer y tercer nivel, y la última entre las de segundo y tercer nivel. Se obtuvo como resultado que el PIT entre las regiones de primer y segundo nivel es fundamentalmente un proceso recíproco, intenso y muy complejo; mientras que el que se configura entre las regiones de primer y tercer nivel, así como entre las de este último y las de segundo nivel, es débil, fragmentado y unidireccional. También se pudo establecer que, si bien las regiones más transmisoras de tecnología son las desarrolladas, estas son a su vez muy receptoras. Así mismo, las de menor nivel de desarrollo son consumidoras netas y poco transmisoras de tecnologías.

Otra reflexión importante de este trabajo es que el reconocimiento de las redes y spillovers recíprocos le agregan contenido a la explicación del PIT entre regiones. Permitió comprender que en este proceso existen diferencias marcadas determinadas por el tipo de participantes que intervienen, que pueden ser agentes desarrollados o, en cambio, agentes de distinto nivel de desarrollo. Aquí se ha mostrado que, en el primer caso, el PIT es más intenso y que supera el $50 \%$ de las relaciones posibles. Mientras que, en el segundo, las relaciones estarán siempre por debajo de este baremo; lo cual indica que el PIT será distinto, operará bajo otras reglas y principios. Aquí se tendrán que considerar las instituciones locales en el PIT; al tiempo que se deben evaluar las diferencias en el impacto de la IED en la productividad de las regiones y no suponer que son semejantes.

En esa dirección, se avanzó hasta mostrar que las regiones no son autosuficientes a la hora de generar todo el conocimiento. Todas tienen, en mayor o menor medida, algún conocimiento que transferir, a la vez que tecnología que recibir. La diferencia radica en los roles, unas son transmisoras netas, mientras que otras son más receptoras. Esto permite inferir que es posible perfeccionar los SRI, potencializando los agentes más competitivos en conocimiento, para hacerlos atractivos para el resto del mundo. 
El reconocimiento de las redes y los spillovers invita a proponer políticas que estimulen estos dos fenómenos en el PIT. Bajo esta lógica se deben diseñar instrumentos que fortalezcan la reciprocidad y transitividad entre los agentes, y formular incentivos que estimulen la organización flexible de los proyectos y contratos de transferencia de tecnología. Esto implica profundizar en el estudio de las redes como formas organizativas, es decir, entender las propiedades, la lógica de los vínculos, el papel de la distancia y el estímulo a la asociación; pues con la comprensión del funcionamiento de las redes se podrán diseñar políticas amplias, incluyentes, globales y colaborativas.

En esta investigación se logró poner en evidencia la importancia del uso del ARS como estrategia metodológica para el análisis de transferencia de tecnología y conocimiento; por tanto, el avance en este trabajo radica en haber podido brindar elementos y herramientas para adentrarse en el estudio del PIT en presencia de redes y spillovers. No obstante, este tipo de investigaciones enfrentan varias limitaciones: como por ejemplo, la disponibilidad de información sobre los spillovers, pues la literatura ha mostrado que se tropieza con problemas cuando se quiere capturar esta clase de externalidades. Otra limitación está asociada al análisis de los vínculos y, por tanto, a la formación de la red; como se sabe, las relaciones entre los SRI se establecen mediante proyectos, contratos puntuales, intercambio de servicios, etc.; el problema radica en la comparabilidad de todas estas formas alternativas de relación y su homogenización.

\section{Referencias}

Albornoz, M., \& López, J. (eds.) (2015). Ciencia, tecnología y universidad en Iberoamérica. Buenos Aires: Eudeba.

Asheim, B., Boschma, R., \& Cooke, P. (2011). Constructing regional advantage: Platform policies based on related variety and differentiated knowledge bases. Regional Studies, 45(7), 893-904.

Balland, P., Boschma, R., \& Frenken, K. (2015). Proximity and innovation: From statics to dynamics. Regional Studies, 49(6), 907-920.

Boschma, R. (2014). Constructing regional advantage and smart specialization: Comparison of two European policy concepts. Scienze Regionali, 1(1), 51-68.

Bozeman, B. (2000). Technology transfer and public policy: A review of research and theory. Research Policy, 29, 627-655.

Bozeman, B., \& Boardman, C. (2014). Assessing research collaboration studies: A framework for analysis. InResearch Collaboration and Team Science(pp. 1-11). Cham, Switzerland: Springer. 
Bradley, S., Hayter, C., \& Link, A. (2013). Models and methods of university technology transfer. Foundations and Trends in Entrepreneurship, 9(6), 571-650.

Caicedo, H. (2012). Análisis del sistema regional de ciencia, tecnología e innovación del Valle del Cauca. Estudios Gerenciales, 28 (ed. especial), 125-148.

Caicedo, H. (2017). Identificación, caracterización y tipología de las regiones globales basadas en el conocimiento: una aproximación desde la coordinación de las políticas de desarrollo.Estudios Socioterritoriales,21, 91-110.

Cantwell, J., \& Glac, K. (2004). La estrategia tecnológica de las empresas multinacionales y el desarrollo de capacidades tecnológicas locales. Cuadernos de Economía y Dirección de la Empresa, 20, 83-102.

Carayannis, E., Campbell, D., Grigoroudis, E., \& Monteiro, S. (2017). Introduction. InS. Monteiro \& E. Carayannis (eds.), The Quadruple Innovation Helix Nexus(pp. 1-38). New York: Palgrave Macmillan.

Casas, R., \& Gortari, R. de (2001).La formación de redes de conocimiento: Una perspectiva regional desde México. Rubí [Barcelona], España: Anthropos. Serie Tecnología, Ciencia, Naturaleza y Sociedad, vol. 11.

Casas, R., Corona, J., Jaso, M., Vera-Cruz, A., Caballero, R., \& Rivera, R. (2013). Construyendo el diálogo entre los actores del sistema de ciencia, tecnología e innovación libro conmemorativo a 10 años de la creación del Foro Consultivo Científico y Tecnológico. México DF: Foro Consultivo Científico y Tecnológico.

Caves, R. (1974). Industrial organization. In J. Dunning (ed.), Economic Analysis and Multinational Enterprise (pp. 115-146). London: Praeger.

Coenen, L., Asheim, B., Bugge, M., \& Herstad, S. (2017). Advancing regional innovation systems: What does evolutionary economic geography bring to the policy table? Environment and Planning C: Politics and Space,35(4), 600-620.

Driffield, N., \& Love, J. (2005). Who gains from whom? Competition and technology sourcing in the foreign-owned sector of UK manufacturing. Scottish Journal of Political Economy, 52(5), 663-686.

Gereffi, G., Humphrey, J., \& Sturgeon, T. (2005). The governance of global value chains. Review of International Political Economy, 12(1), 78-104.

Gertler, M., \& Levitte, Y. (2003). Local nodes in global networks: The geography of knowledge flows in biotechnology innovation. Documento presentado en la Conferencia DRUID Summer. Elsinore.

Giudice, M. del, Carayannis, E., \& Maggioni, V. (2017). Global knowledge intensive enterprises and international technology transfer: emerging perspectives from a quadruple helix environment. Journal of Technology Transfer, 42(2), 229-235. 
Godin, B. (2006). The linear model of innovation: The historical construction of an analytical framework.Science, Technology, and Human Values,31(6), 639-667.

Greenacre, M. (2008). La práctica del análisis de correspondencias. Bilbao: Fundación BBVA. Hanneman, R. (2000). Introducción a los métodos del análisis de redes sociales. Recuperado el 20 de agosto de 2017, de https://goo.gl/Jc3QRY

Huggins, R., \& Izushi, H. (2008). Benchmarking the knowledge competitiveness of the globe's high-performing regions: A review of the World Knowledge Competitiveness Index.Competitiveness Review: An International Business Journal, 18(1/2), 70-86.

Huggins, R., Izushi, H., Prokop, D., \& Thompson, P. (2014). The global competitiveness of regions. London: Routledge.

Keeble, D., \& Wilkinson, F. (2017).High-technology clusters, networking and collective learning in Europe. London: Routledge.

Kline, S., \& Rosenberg, N. (1986). Chain-linked model of innovation. In R. Landau \& N. Rosenberg (eds.), An overview of innovation: The positive sum strategy. Washington DC: National Academy Press.

Llisterri, J., Pietrobelli, C., \& Larsson, M. (eds.) (2011). Los sistemas regionales de innovación en América Latina. Washington DC: BID.

Maskell, P., Bathelt, H., \& Malmberg, A. (2006). Building global knowledge pipelines: The role of temporary clusters. European planning studies,14(8), 997-1013.

Morgan, K. (2017). Nurturing novelty: Regional innovation policy in the age of smart specialisation.Environment and Planning C: Politics and Space,35(4), 569-583.

Narula, R. (2010). Much ado about nothing, or sirens of a brave new world? MNE activity from developing countries and its significance for development. Paris: OCDE.

Narula, R., \& Michel, J. (2009). Reverse knowledge transfer and its implications for European policy. Maastricht Economic and Social Research and Training Centre on Innovation and Technology. Working Paper Series/United Nations University, UNU-MERIT, n. 35.

Narula, R., \& Pineli, A. (2017). Multinational enterprises and economic development in host countries: What we know and what we don't know. In G. Giorgioni (ed.), Development Finance(pp. 147-188). London: Palgrave Macmillan.

OCDE (2012). Promoting Growth in All Regions. OECD Publishing. Recuperado el 20 de agosto de 2017, de http://dx.doi.org/10.1787/9789264174634-en

Pietrobelli, C., \& Rabellotti, R. (2011). Global value chains meet innovation systems: Are there learning opportunities for developing countries? World Development, 39(7), 1261-1269.

Ramírez, J., Silva, I., \& Cuervo, L. (2009).Economía y territorio en América Latina y el Caribe: Desigualdades y políticas. Serie Libros de la CEPAL, vol. 99. Chile: CEPAL - ONU. 
Ruscheweyh, R., Deppe, M., Lohmann, H., Wersching, H. et ál. (2013). Executive performance is related to regional gray matter volume in healthy older individuals. Human Brain Mapping,34(12), 3333-3346.

Sassen, S. (2001). Global cities and developmentalist states: How to derail what could be an interesting debate: A response to Hill and Kim.Urban Studies,38(13), 2537-2540.

Sassen, S. (2016).Global networks, linked cities. London: Routledge.

Schmidt, T., \& Sofka, W. (2009). Liability of foreignness as a barrier to knowledge spillovers: Lost in translation? Journal of International Management, 15(4), 460-474.

Storper, M. (1995). The resurgence of regional economies, ten years later the region as a nexus of untraded interdependencies.European Urban and Regional Studies,2(3), 191-221.

Storper, M., \& Venables, A. (2004). Buzz: face-to-face contact and the urban economy.Journal of Economic Geography, 4(4), 351-370.

Suanes, M., \& Roca-Sagalés, 0. (2015). Inversión extranjera directa, crecimiento económico y desigualdad en América Latina. Trimestre Económico,82(327), 675-706.

Taylor, P. (2004).World city network: A global urban analysis. London: Routledge.

Yi, J., Chen, Y., Wang, C., \& Kafouros, M. (2015). Spillover effects of foreign direct investment: How do Region-Specific Institutions Matter?Management International Review,55(4), 539-561. 